\title{
Validation of Nonrigid Image Registration Using Finite-Element Methods: Application to Breast MR Images
}

\author{
Julia A. Schnabel*, Christine Tanner, Andy D. Castellano-Smith, Andreas Degenhard, Martin O. Leach, \\ D. Rodney Hose, Derek L. G. Hill, Member, IEEE, and David J. Hawkes
}

\begin{abstract}
This paper presents a novel method for validation of nonrigid medical image registration. This method is based on the simulation of physically plausible, biomechanical tissue deformations using finite-element methods. Applying a range of displacements to finite-element models of different patient anatomies generates model solutions which simulate gold standard deformations. From these solutions, deformed images are generated with a range of deformations typical of those likely to occur in vivo. The registration accuracy with respect to the finite-element simulations is quantified by co-registering the deformed images with the original images and comparing the recovered voxel displacements with the biomechanically simulated ones. The functionality of the validation method is demonstrated for a previously described nonrigid image registration technique based on free-form deformations using B-splines and normalized mutual information as a voxel similarity measure, with an application to contrast-enhanced magnetic resonance mammography image pairs. The exemplar nonrigid registration technique is shown to be of subvoxel accuracy on average for this particular application. The validation method presented here is an important step toward more generic simulations of biomechanically plausible tissue deformations and quantification of tissue motion recovery using nonrigid image registration. It will provide a basis for improving and comparing different nonrigid registration techniques for a diversity of medical applications,
\end{abstract}

Manuscript received September 12, 2001; revised October 23, 2002. The work on biomechanical tissue modeling using ANSYS was supported by the EPSRC. The work of J. A. Schnabel was supported by EasyVision Advanced Development, Medical Imaging Information Technology (MIMIT), Philips Medical Systems, Best, The Netherlands. The work of C. Tanner was supported by the EPSRC under Grant GR/M52779 and Grant MIAS-IRC. The work of A. D. Castellano-Smith was supported by the EPSRC under Grant GR/M47294. The work of A. Degenhard was supported by the EPSRC under Grant GR/M52762. The Associate Editor responsible for coordinating the review of this paper and recommending its publication was M. Giger. Asterisk indicates corresponding author.

*J. A. Schnabel is with the Computational Imaging Sciences Group, Division of Imaging Sciences, Guy's, King's and St. Thomas' School of Medicine, King's College London, London SE1 9RT, U.K. (e-mail: julia.schnabel@kcl.ac.uk).

C. Tanner, A. D. Castellano-Smith, D. L. G. Hill, and D. J. Hawkes are with the Computational Imaging Sciences Group, Division of Imaging Sciences, Guy's, King's and St. Thomas' School of Medicine, King's College London, London SE1 9RT, U.K.

A. Degenhard was with the CRC Clinical MR Research Group, The Institute of Cancer Research and the Royal Marsden NHS Trust, Sutton, Surrey SM2 5PT, U.K. He is now with the Faculty of Physics, University of Bielefeld, 33615 Bielefeld, Germany.

M. O. Leach is with the CRC Clinical MR Research Group, The Institute of Cancer Research and the Royal Marsden NHS Trust, Sutton, Surrey SM2 5PT, U.K.

D. R. Hose is with the Clinical Sciences Division, Department of Medical Physics and Clinical Engineering, Royal Hallamshire Hospital, University of Sheffield, Sheffield S10 2JF, U.K.

Digital Object Identifier 10.1109/TMI.2002.808367 such as intrasubject tissue deformation or motion correction in the brain, liver or heart.

Index Terms-Biomechanics, breast MR imaging, finite element methods, image registration, validation.

\section{INTRODUCTION}

MAGE registration describes the process of establishing spatial correspondence between features in an image pair, or a dynamic or temporal sequence of images, in order to relate them for diagnosis, inspection of homologous positions, or temporal monitoring. The images might be acquired using the same or different imaging modalities, and can also be aligned to a computer model, or to locations in physical space for image guidance. Feature alignment is described by a transformation, which, for rigid-body registration, describes differences in global patient positioning. For nonrigid registration, the transformation explains additional deformations due to soft tissue properties, surgical intervention, temporal changes due to tumor growth or radiotherapy treatment, and morphological differences between individuals. Also, nonrigid registration can compensate for geometric image distortion caused by the acquisition technique. There is a consensus in the literature that registration is needed to compensate for patient positioning and deformation. Various registration algorithms have been proposed, surveys of which can be found in [1]-[5]. Prior to clinical use, registration techniques need to be validated. However, validation of registration performance suffers from the lack of knowledge as to if, how much, and where patient movement has occurred between and even during scanning procedures, and whether such movement affects the clinical usefulness of the data. To maintain clinical usefulness, and inherently improve patient treatment and health care, it is, therefore, mandatory to ensure that registration is successful. How the success of medical image registration can be assessed and failure can be detected is laid out in a recent survey by Fitzpatrick [6], and will be discussed with an emphasis on validation of nonrigid registration in the following.

As a first step, a registration method can be assessed in an independent evaluation in the absence of a ground truth. An initial visual inspection allows for a qualitative assessment of registration performance, which can be complemented by quantitative checks for robustness and consistency. The former establishes the measurement precision by testing the bias sensitivity when adding noise or choosing different starting estimates [7]. 
The latter assesses the capability of a registration technique to find circular transformations based on a registration circuit, but can be sensitive to bias and may not be applicable to noninvertible transformations generated by many nonrigid registration methods. Nonetheless, consistency checks have been successfully used for intramodality rigid body registration applications, e.g., for serial magnetic resonance (MR) imaging of the brain [8].

As a second step, the registration outcome can be judged and ranked by expert observers using visual assessment techniques in a large study. This can involve the inspection of subtraction images, contour or segmentation overlays, alternate pixel displays, or viewing anatomical landmarks. These approaches have been applied to rigid registration [9], and since they involve inspection of the entire volume domain of the image pair, can be extended to nonrigid registration [10]. Visual assessment is an important step toward clinical acceptance and routine use of a registration method, but may be compromised for nonrigid registration by locally implausible deformations which may not be readily picked up by observers [11].

As a third step, quantitative measures assessing the accuracy of a registration method can be performed. Accuracy, however, can only be measured if a ground truth is available. Maintz and Viergever [3] argue that if such ground truth techniques existed, they should be used for the registration in the first place. Nonetheless, registration accuracy can be studied while keeping the error bounds in mind. The main approach for estimating accuracy involves establishing a gold standard. For example, the retrospective registration evaluation project [12] used skull-implanted markers in patients undergoing brain surgery to derive a gold standard transformation for multimodality rigid-body image registration of the head to compare different established registration methods. For nonrigid registration validation, extrinsic markers could be attached to the skin surface or implanted into deformable tissue. Skin markers have the advantage of not being invasive, but suffer from movement related to skin mobility, and from being far from more internal, relevant anatomical structure. Implanted markers are highly invasive and, therefore, can only be used if a patient is undergoing surgery. At any rate, such markers may not provide a sufficiently dense displacement field for nonrigid registration validation. This is also true for intrinsic markers like anatomical landmarks, which are often too sparse and too difficult to localize accurately. As an alternative, physical phantoms or cadavers with densely distributed markers could be used, but they cannot be easily deformed in a controlled manner, nor are accuracy measures obtained in vitro readily transferable to in vivo applications. As an alternative to markers, a gold standard registration system can be used to simulate "ground truth" transformations against which transformations obtained from registration can be compared [6]. For nonrigid motion simulation, the most common approach is to displace a set of landmarks and interpolate a dense displacement map using thin-plate splines or other interpolants (e.g., [13]). The landmarks may be anatomical or geometric, or corresponding to intersections of a regular grid superimposed on the image. Such simulations commonly ignore the underlying tissue properties, which may lead to physically and biomechanically unrealistic deformation simulations. Moreover, in many cases the same or a similar displacement interpolant is used in the subsequent registration process, which could introduce a bias of the validation toward the registration method.

Finally, there are more practical validation issues to consider. For example, Maintz and Viergever [3] list resource requirements and algorithmic complexity, which refer to preparations required for the registration (such as segmentation of structures, or landmark extraction), and computational time constraints for use in clinical practice, respectively. Automated registration methods can help to fulfill such requirements, and, with computing power improving, the run-time of registration algorithms continues to decrease.

The validation steps described above highlight a number of unresolved research issues for nonrigid registration validation. In this paper, we address the simulation of gold standard deformations to measure nonrigid registration accuracy. We present a novel biomechanically motivated validation methodology which is based on modeling tissue properties and simulating tissue deformations using finite-element methods (FEMs). Earlier work on this technique was presented by us in [14]. This simulation comprises a range of physically plausible tissue deformations which are most likely to happen in clinical practice and, hence, provides more insight into the reliability of the registration, i.e., the behavior of the algorithm to be expected in a real clinical setting for reasonable clinical input [3]. The accuracy of the registration can be established via a complete, dense map of simulated voxel displacements. We apply this validation method to a previously described nonrigid registration algorithm by Rueckert et al. [15] for contrast-enhanced (CE) MR mammography. Although the validation method is applied here to a specific algorithm and clinical application, it describes a generic methodology for validating nonrigid registration algorithms. This methodology requires FEM modeling of the target anatomy but may be applied to a variety of registration tasks, e.g., motion or deformation correction in the brain, liver or heart. The purpose of this paper is to present the concept of the validation framework using an exemplar registration technique and application. It is designed for intrasubject registration tasks, where tissue deformation with respect to a subject's anatomy and an assumed material model can be simulated using biomechanical modeling. Intersubject registration validation using deformation simulations is an even more challenging task, as this would also require a model of anatomical variability, which has so far only been attempted using statistical models of variation [16], [17].

The remainder of this paper is organized as follows: Section II briefly describes the exemplar nonrigid registration method used, and Section III describes the breast tissue modeling using FEMs. Section IV presents the simulation of biomechanical deformations in breast tissue, and Section V summarizes the registration accuracy measurements obtained. Finally, Section VI summarizes the presented validation methodology, outlines the potential for further improvement, and concludes this paper.

\section{NONRIGID REGISTRATION}

In this paper, we use as an exemplar registration method a nonrigid registration algorithm which was previously developed by Rueckert et al. [15] for motion compensation in three-dimensional (3-D) CE MR mammography. The algorithm is based 
on free-form deformations using B-splines, and uses normalized mutual information as a voxel-similarity measure [18]. It models global patient motion using an affine transformation, followed by modeling local motion by manipulating an underlying mesh of B-spline control points. The combined global and local motion model at each image point $\mathbf{x}=(x, y, z)$ is expressed as

$$
\mathbf{T}(\mathbf{x})=\mathbf{T}_{\text {global }}(\mathbf{x})+\mathbf{T}_{\text {local }}(\mathbf{x})
$$

The flexibility and computational complexity of the local motion model is related to the choice of control point spacing. The algorithm makes no assumption about the underlying material properties of the different tissue types in the breast.

In a visual assessment study, the algorithm was shown to significantly improve the image quality of the subtraction images for a large MR mammography database [10]. For this application, patient movement as well as tissue deformation often occur during dynamic scanning due to patient reaction to contrast injection, contraction and relaxation of the pectoral muscles, as well as movement against the scanner radio-frequency (RF) coil. As tumors are often only clearly seen on subtraction images, any misalignment may render enhanced tumors indistinguishable from surrounding bright motion artefacts. Recently, we have found that this algorithm can cause volume changes in regions of enhanced lesions in MR mammography [11]. These volume changes may occur due to the similar intensity of fatty tissue and CE fibroglandular tissue, but are physically unlikely given the incompressibility of the breast tissue, and the short acquisition time of the dynamic image sequence. There is, therefore, a need to further investigate the behavior and accuracy of this algorithm using simulations of patient motion in CE MR mammography.

\section{Finite-ElEMENT Modeling OF THE BREAST}

\section{A. Background}

The modeling of biomechanical tissue properties has gained considerable interest in a range of clinical and research applications. FEMs can be used to model the interrelation of different tissue types by applying displacements or forces. This can help to predict mechanical or physical deformations during surgical procedures, and to derive and quantify tissue properties from observed deformations. For example, FEMs for brain modeling have been investigated for model updating of image guided surgery procedures [19]-[21], for integration into physically based nonrigid registration methods [22], and for simulation of brain shift in interventional MR imaging [23]. For mammography, FEMs have been explored for predicting mechanical deformations during biopsy procedures [24], for simulating compressions similar to X-ray mammography in MR mammography [25], for improving and testing the reconstruction of elastic properties in elastography [26]-[28], and for modality-independent elastography with application to breast imaging [29].

\section{B. Materials}

From a database of previously acquired dynamic sequences of Gadolinium DTPA (Gd-DTPA)-enhanced MR mammography volumes of 42 patients with histologically confirmed
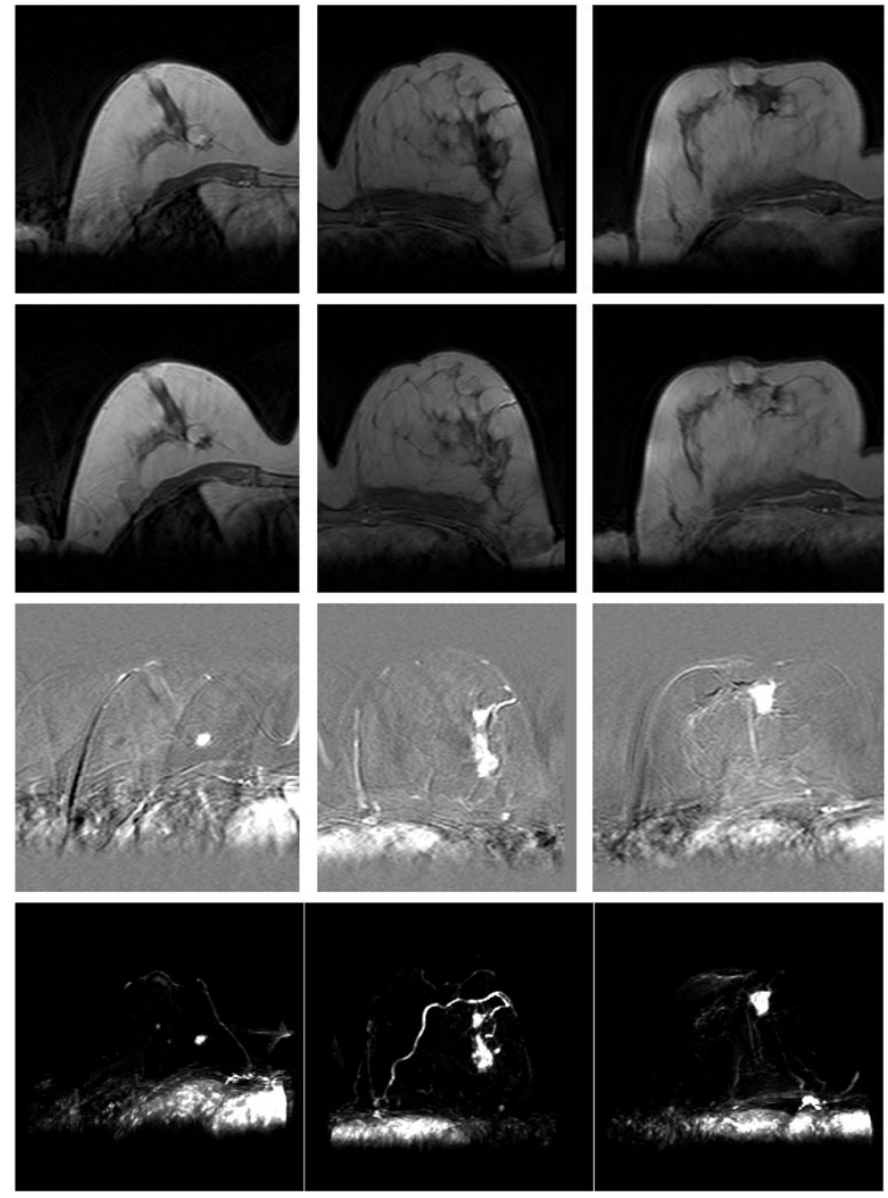

Fig. 1. Two-dimensional example slices, subtractions, and maximum intensity projections (MIPs) of subtraction volumes for three MR breast volumes. (from left to right) Patient cases 1-3. (from top to bottom) Precontrast image, postcontrast image, subtraction image, and MIP.

carcinoma, three patient cases were selected. All data were acquired on a Philips 1.5-T Gyroscan ACS2 using a fast 3-D gradient echo sequence with $\mathrm{TR}=12 \mathrm{~ms}, \mathrm{TE}=5 \mathrm{~ms}, 35^{\circ}$ flip angle, 350-mm field of view (FOV), and axial (transversal) slice orientation. A dynamic sequence of one scan before, and five scans after contrast injection of $0.2-\mathrm{mmol} \mathrm{Gd}-\mathrm{DTPA} / \mathrm{kg}$ of body weight at temporal intervals of $1 \mathrm{~min}$ was acquired. For the purpose of this paper, we have selected the precontrast scan and the second postcontrast scan of the three patients. The images have dimensions of $256 \times 256 \times 25$ voxels with an in-plane voxel size of $1.37 \mathrm{~mm} \times 1.37 \mathrm{~mm}$ (cases 1 and 2) and $1.48 \mathrm{~mm} \times 1.48 \mathrm{~mm}$ (case 3 ), all with $4.2-\mathrm{mm}$ slice thickness. From each data set, we have extracted a volumetric cuboid region of interest (ROI) containing one breast for each patient. Both the anatomy and pathology of the selected cases are substantially different, with the overall breast volume ranging from $0.66 \cdot 10^{6} \mathrm{~mm}^{3}$ (case 1) to $1.23 \cdot 10^{6} \mathrm{~mm}^{3}$ (case 3), with tumor volumes between $235.52 \mathrm{~mm}^{3}$ (case 1) and 3061.75 $\mathrm{mm}^{3}$ (case 3).

The three cases were selected because unlike the great majority of cases, there was very little subject motion between acquisitions. This was confirmed in the visual assessment study [10], where nonrigid registration did not significantly improve the subtraction images for cases 2 and 3, and only improved it to a small degree for case 1 near the skin surface. Fig. 1 
shows two-dimensional (2-D) example slices through the ROIs of the image pairs and image subtractions, as well as maximum intensity projections (MIPs) through the subtraction volumes. From the subtraction images as well as the MIPs one can appreciate the negligible amount of motion between precontrast and postcontrast scans within the breast tissue, and motion artefacts due to breathing and cardiac motion at the chest wall. The tumors and some enhanced blood vessels become clearly visible within the subtraction images and MIPs. Nonrigid registration confirmed almost no motion for the original image pairs: mean tissue displacements within the breast tissue, excluding the tumor region, were estimated to be within $1 \mathrm{~mm}$ (case 1), $0.36 \mathrm{~mm}$ (case 2), and $0.55 \mathrm{~mm}$ (case 3). Within the tumor regions, mean tissue displacements of $0.45 \mathrm{~mm}$ (case 1), $0.68 \mathrm{~mm}$ (case 2), and $0.40 \mathrm{~mm}$ (case 3) were found. Having selected image pairs with only little motion enables us to simulate motion between the original, uncorrected precontrast and postcontrast scans.

\section{Model Construction}

For the construction of finite-element models for the three cases, the first step is to obtain tissue segmentations. For this, the ANALYZE software package [30] was used to segment fatty and fibroglandular tissue in the CE images. The tumors were segmented in the subtraction images, which was made possible due to the negligible amount of motion between scans.

As the second step, we have constructed for the three cases isotropic, linear, and nearly incompressible elastic models incorporating skin surface, fat, fibroglandular, and tumorous tissue. For this purpose, we have performed 3-D triangulations of the tumors and the overall breast tissue (excluding the tumorous tissue) using standard marching cubes and decimation techniques provided by the Visualization Toolkit [31], with minimal edge lengths of $4.2 \mathrm{~mm}$ (corresponding to the slice thickness) for tumorous tissue and $8.4 \mathrm{~mm}$ for the remaining tissues. Using the ANSYS FEM software package, ${ }^{1}$ the triangulations were meshed into isoparametric tetrahedral structural solids (elements), having four corner FEM nodes ${ }^{2}$ and an additional FEM node in the middle of each edge for a quadratic displacement behavior. Each FEM element was labeled corresponding to the underlying tissue type. Skin was modeled by adding 1-mm-thick triangular shell elements consisting of six FEM nodes onto the surface of the fatty tissue. The models contain 40172 (case1), 117436 (case 2), and 118278 (case 3) FEM nodes, arranged in 30841 (case 1), 89094 (case 2), and 89944 (case 3) elements, including the surface shells. Each FEM node has three associated degrees of freedom which define translational displacement in the $x, y$, and $z$ directions.

As the third and final step in the model construction, material properties for the different tissue types were chosen from the literature. The Young's moduli (stress-strain relations) of the different tissues were set to $1 \mathrm{kPa}$ for the fatty tissue, $10 \mathrm{kPa}$ for the fibroglandular tissue, and $16.5 \mathrm{kPa}$ for the tumorous carcinoma [32]. A Young's modulus of $88 \mathrm{kPa}$ was chosen for

\footnotetext{
${ }^{1}$ Available: http://www.ansys.com

${ }^{2}$ To avoid any confusion with anatomical tissue nodes in the pectoral breast region, or the B-spline control points used in the registration, we will refer to the nodes of the FEM elements as FEM nodes in the remainder of this paper.
}
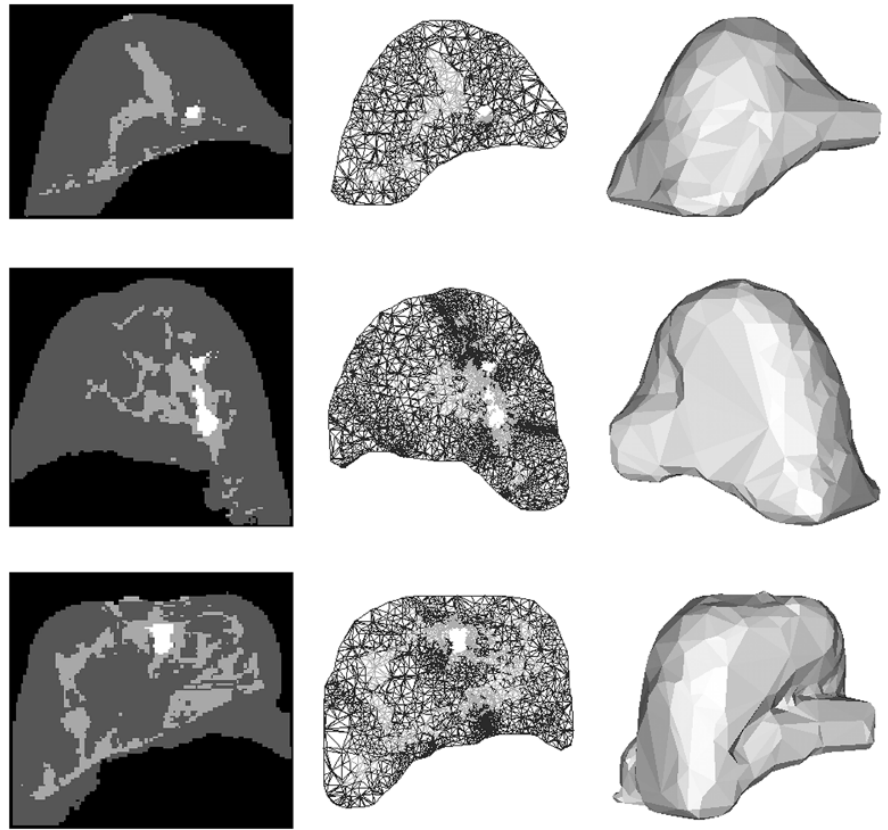

Fig. 2. Breast segmentations and FEM model renderings for patient cases shown in Fig. 1. (from top to bottom) Patient cases 1-3. (from left to right) Example slices for segmentations into fat (dark grey), fibroglandular tissue (light grey), and tumor (white); wire-frame cuts through FEM models with material labels in the same color code; mesh surface renderings of FEM models. The wire-frame cuts show the higher mesh resolution within tumor locations.

the skin, representing a linear approximation of the nonlinear stress-strain curve for abdominal skin parallel to the craniocaudal median investigated by Park [33] for strains up to $30 \%$. For near-incompressibility of the tissue, the Poisson's ratio was set to 0.495 .

The aim of this study is to obtain approximate breast models which can produce physically plausible deformations, rather than to build optimal, patient-specific models. This allows the generation of a generic image class for registration validation. For more realistic breast modeling, patient-specific model parameters would need to be determined from in vivo elastographic measurements. However, the models described above can be varied further with respect to material properties, nonlinear or even anisotropic tissue characteristics, in order to simulate different patient characteristics. Fig. 2 shows example slices through the tissue segmentations, material-labeled wireframe renderings as well as surface renderings of the three breast models.

\section{Simulation of Gold Standard Breast Deformations}

\section{A. Finite-Element Model Solutions}

In a previous study involving radiologists, it was found that due to the confinement of the breast within the scanner RF coil, tissue motion of up to $15 \mathrm{~mm}$ in extreme cases was observed between the acquisition of precontrast and postcontrast image volumes [10], but for the great majority of the cases deformations were found to be $10 \mathrm{~mm}$ or less. Consequently, FEM boundary conditions using initial surface displacements of approximately $10 \mathrm{~mm}$ were applied to the three models presented above. The models were subsequently solved using a preconditioned conjugate gradient solver of the ANSYS FEM package. To each 

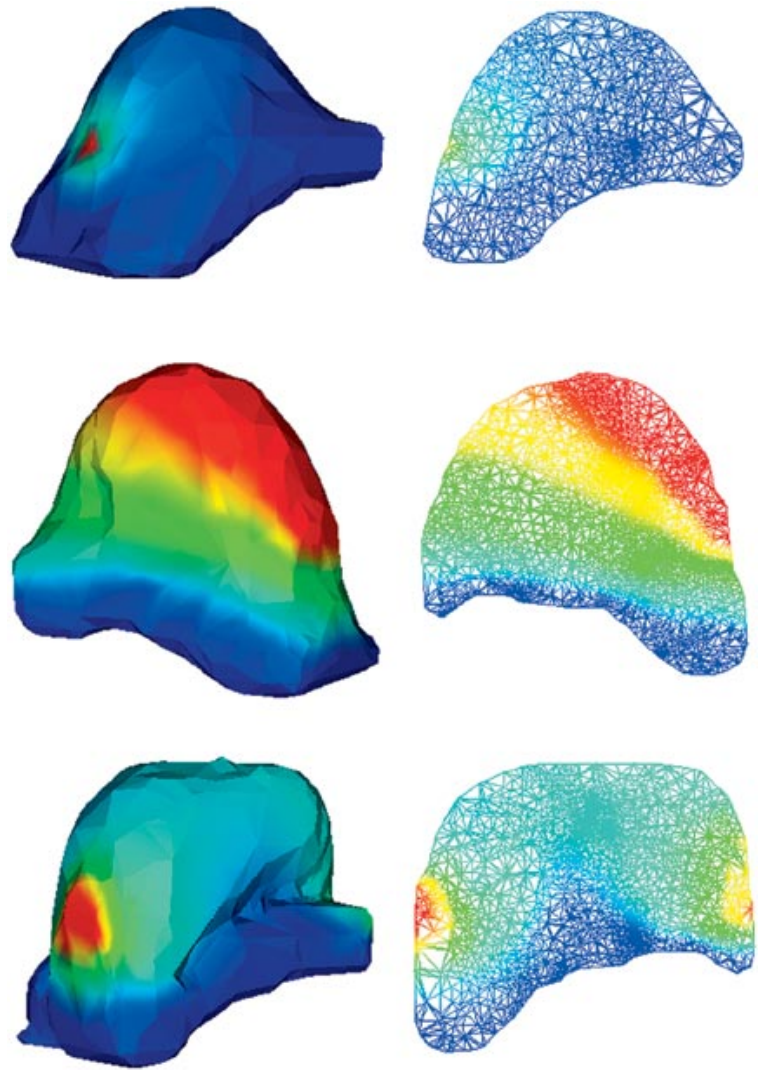

Fig. 3. Surface and wire-frame renderings of example solutions for models shown in Fig. 2. (from top to bottom) Patient cases 1-3, for point puncture (case 1), regional displacement (case 2), and two-sided contact (case 3). (left) Surface renderings of deformed meshes. (right) Wire-frame cuts through deformed meshes. Deformation magnitudes are mapped using rainbow color coding, with dark blue corresponding to $0 \mathrm{~mm}$, and dark red corresponding to 10-mm deformation magnitude.

breast model, the following four deformation types characteristic for real patient acquisitions were applied.

- Regional displacement simulates a uniform surface displacement by translating a set of surface FEM nodes.

- Point puncture displaces a single surface FEM node which simulates a very localized displacement, e.g., as occurring during a biopsy without any breast fixation.

- One-sided contact displaces surface FEM nodes on one side onto a plane, which simulates the deformation of the breast when moving against the scanner RF coil, assuming no sliding on the plane itself.

- Two-sided contact analogously models the deformation when the breast is fixed at both sides, by displacing surface FEM nodes onto a plane on each side.

Thus, we have obtained 12 deformation simulations (three breast models with four deformation simulations each). For more exhaustive deformation simulations, additional simulations can be performed by varying the type, magnitude, and locality of the boundary conditions. In all simulations, the FEM nodes adjacent to the deep pectoral fascia have been fixed, assuming no movement of the pectoral muscle and pectoral fascia. Fig. 3 shows surface renderings of and wire-frame cuts through the patient models for example FEM solutions, with local deformation magnitudes mapped in rainbow color coding.

\section{B. FEM Displacements}

Each FEM solution yields a displacement vector $\mathbf{u}_{i}=$ $(d x, d y, d z)$ at each FEM node within the model. The average displacement of the whole breast volume and within individual tissue compartments can be obtained by integrating over all displacement vectors

$$
\mathcal{E}_{\text {node }}=\frac{1}{N} \sum_{i=1}^{N}\left\|\mathbf{u}_{i}\right\|
$$

where $N$ is the number of FEM nodes in the model or of individual tissue compartments.

For an image voxel (or subvoxel) location $\mathbf{x}=(x, y, z)$ not coinciding with a FEM node location, but lying anywhere within a FEM element, a numerically exact displacement can be interpolated by weighting the element's ten FEM node displacements $\mathbf{u}_{j}$ by their quadratic shape function $S(j)$ [34]

$$
\mathbf{u}(\mathbf{x})=\sum_{j=1}^{10} S(j) \mathbf{u}_{j} .
$$

Hence, a dense displacement interpolation for all image voxels $\mathbf{x}_{i}$ can be obtained, and integrated over all voxel locations within the breast volume or within individual tissue compartments to find the average displacement

$$
\mathcal{E}_{\text {shape }}=\frac{1}{n} \sum_{i=1}^{n}\left\|\mathbf{u}\left(\mathbf{x}_{i}\right)\right\|
$$

where $n$ is the number of voxels over which this measure is computed.

Fig. 4 shows the average and maximum node and interpolated voxel displacements for all patient solutions within the whole breast volume as well as only within the tumorous tissue. The maximum displacements are around $10 \mathrm{~mm}$, mostly occurring in fatty tissues close to the skin surface. A higher mean displacement within the tumors occurs for cases 2 and 3, where the tumors are located close to the initially displaced skin surface.

\section{Interpolation of FEM Solutions}

In order to simulate deformation in the images for registration application, the images need to be transformed using the dense displacement field obtained from each of the FEM solutions. In earlier work [14], we have used a scattered data interpolation technique by Lee et al. [35] based on a multilevel B-spline hierarchy whose sum approaches the desired interpolation, and which can be reformulated into one equivalent B-spline interpolator. One advantage of using such an interpolator is that simulated deformations within the finite-element mesh can be appropriately blended off at the mesh surface and, thus, discontinuities in the deformation field can be reduced. This is particularly important if the mesh surface, due to triangulation and decimation, has relaxed and may not fully fit the original breast volume. The major disadvantage of this approach, however, is that due to the approximating nature of B-splines, a small residual error at the FEM node locations may remain, and that a favorable bias toward spline-based registration techniques could be introduced. 


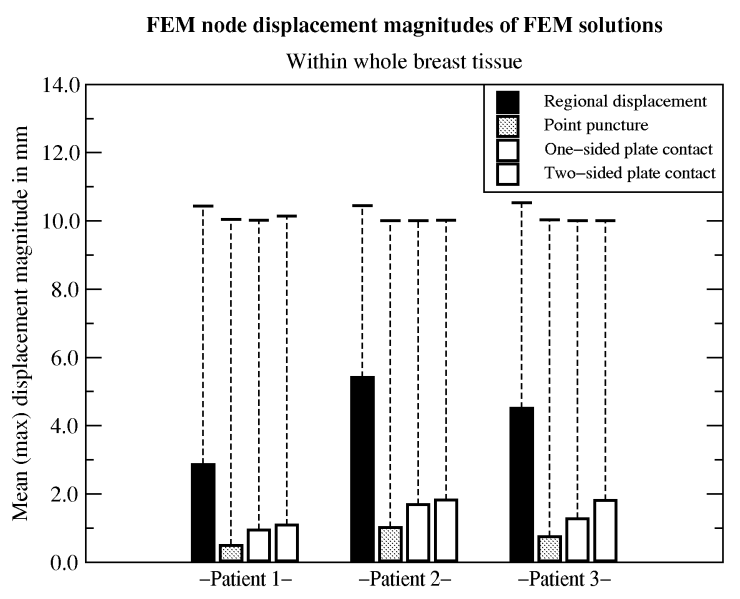

Interpolated tissue displacement magnitudes of FEM solutions

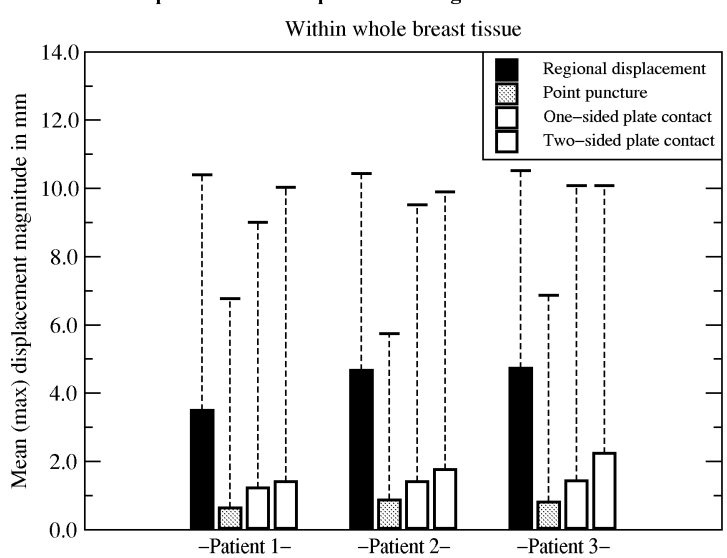

FEM node displacement magnitudes of FEM solutions

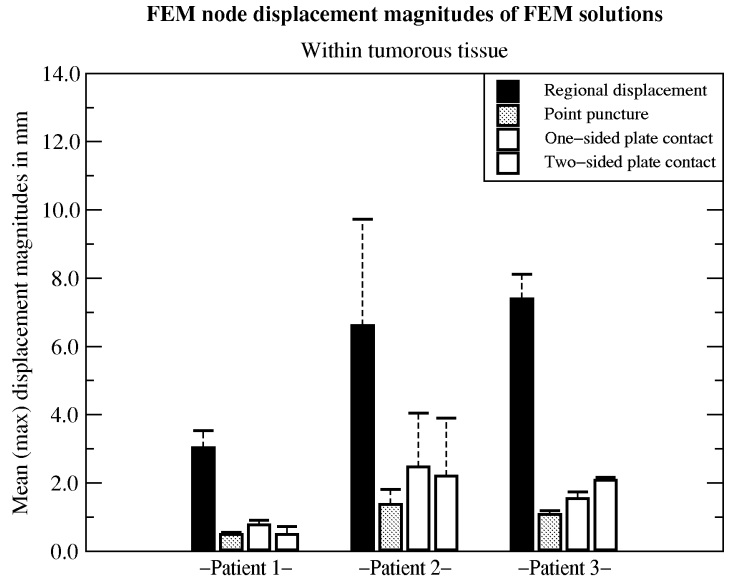

Interpolated tissue displacement magnitudes of FEM solutions Within tumorous tissue

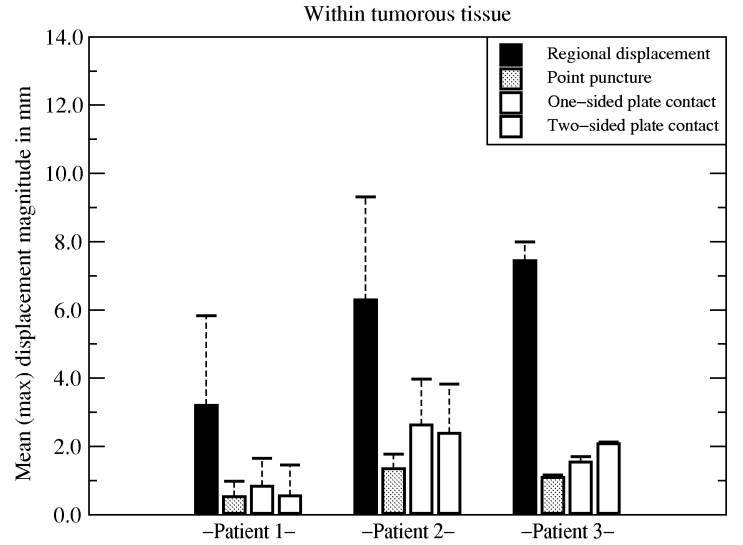

Fig. 4. Mean and maximum FEM node and interpolated voxel displacements of different FEM solutions for the three patient cases, computed within whole breast tissue (left) and within tumorous tissue (right). (top) FEM node displacements. (bottom) FEM shape-interpolated voxel displacements.

To avoid any simulation inconsistencies, we instead employ the accurate FEM shape interpolation defined in (3). This interpolation removes any residual bias of the validation method toward the motion model used by the registration method. Based on the dense displacement fields within the breast tissue, the image intensities are interpolated using a truncated sinc interpolation kernel [36]. It is important to note that since by definition no voxel displacements occur at locations outside of the model, these locations need to be masked out in the deformed images and excluded from any further processing and analysis. For this reason, and to avoid any further intensity interpolation of the simulated image volumes during registration, the FEM deformed images become the reference (or target) image volumes, and the original, undeformed and unmasked images are the transform (or source) image volumes for the subsequent registration process. If an image volume is to be registered against a FEM deformed version of itself, the noise field of the FEM deformed image is changed by adding Rician distributed noise with a standard deviation corresponding to the noise distribution in the original image background.

The original postcontrast images of the three patient cases were deformed with respect to the four FEM solutions and associated dense displacement interpolations. Fig. 5 shows example slices through the FEM deformed postcontrast images of the three patients for the three example solutions shown in Fig. 3.
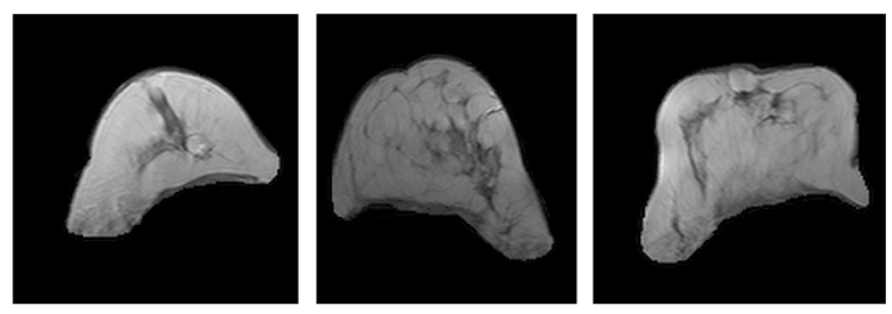

Fig. 5. Slices of example FEM deformation simulations of postcontrast image volumes. (from left to right) Patient cases $1-3$, for point puncture (case 1), regional displacement (case 2), and two-sided contact (case 3). Compare with original postcontrast image slices in Fig. 1.

\section{EXPERIMENTS AND RESULTS}

\section{A. Experiments}

To demonstrate the potential of the proposed validation scheme, we have applied it to test the exemplar nonrigid registration algorithm described in Section II with a control point resolution of $10 \mathrm{~mm}$ as a sensible choice, given the expected maximum displacements of $10 \mathrm{~mm}$ imposed by the FEM boundary conditions. Another motivation for using this particular resolution is that it was successfully used in the visual assessment study on the same data [10].

We have chosen to deform only the postcontrast images in order to first assess whether the deformation can be retrieved 
by registering the original postcontrast images to a deformed version of themselves. A more realistic setting, where patient motion or deformation has occurred between precontrast and postcontrast scans, is then simulated by registering the original precontrast images to the FEM deformed postcontrast images. This approach is based on the reasonable assumption that for the selected patient cases, actual motion between the original precontrast and postcontrast images was negligible.

The registration accuracy can then be assessed either at the FEM node positions [using $\mathbf{u}_{i}$ of (2)], or over the entire interpolated displacement field within the warped breast volumes [using $\mathbf{u}\left(\mathbf{x}_{i}\right)$ of (4)]. The latter is more consistent in the sense that it provides a denser sampling of displacements for accuracy measurements, in view of the scattered distribution of the FEM nodes. The residual registration error for a given transformation $\mathbf{T}$ [as defined by (1)] is then defined for all tissue locations or subsets thereof as

$$
\mathcal{E}_{R}=\frac{1}{n} \sum_{i=1}^{n}\left\|\left(\mathbf{x}_{i}+\mathbf{u}\left(\mathbf{x}_{i}\right)\right)-\mathbf{T}\left(\mathbf{x}_{i}\right)\right\|
$$

where $n$ is the number of voxels $\mathbf{x}_{i}$ at which the error measure is calculated.

\section{B. Postcontrast-to-Postcontrast Image Registration Accuracy}

Fig. 6 illustrates example 2-D slices for the three patient cases through the subtracted images volumes before and after registration of the postcontrast images to the FEM deformed versions of themselves, with the difference of artificially added Rician distributed noise in the warped images. Before registration, a considerable amount of deformation imposed by the FEM solutions near to the skin surface, and to a lesser degree within the breast tissues, can be observed. Ideally, after registration the subtraction images should contain only the added noise. Fig. 6 shows that the deformations are mostly recovered within the breast tissue after registration, with remaining localized misregistrations only near the skin surface, and at the edge of the FOV.

The residual average and maximum registration errors according to (5) for the postcontrast registration experiment are shown in the top row of Fig. 9. The average registration error is about $0.4 \mathrm{~mm}$, with maximum errors between $2.4 \mathrm{~mm}$ and up to $10 \mathrm{~mm}$. These maximum errors were found to be very localized near the edge of the FOV and the displaced skin surface. When investigating the registration accuracy within the tumorous tissues separately, the average error for the postcontrast registration experiment is very low and lies around $0.17 \mathrm{~mm}$, with only a small maximum error of up to $0.45 \mathrm{~mm}$.

\section{Precontrast-to-Postcontrast Image Registration Accuracy}

The clinically more realistic and relevant experiment of registering the original precontrast images to FEM deformed postcontrast images investigates how well simulated patient motion between precontrast and postcontrast acquisitions can be recovered by registration. The subtraction slices in the top row of Fig. 7 show that the tumors are barely visible before registration, and cannot be clearly distinguished from the surrounding bright motion artefacts. However, after registration, these motion artefacts have been mostly removed, apart from localized areas near the skin surface (bottom row of Fig. 7). Fig. 8 shows additional
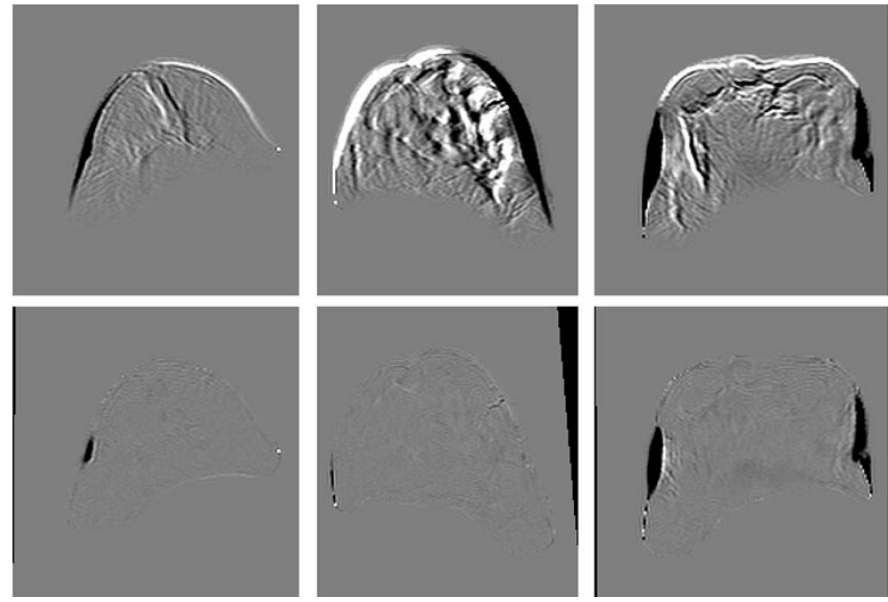

Fig. 6. Example subtraction slices of original postcontrast images from example FEM deformation simulations before (top) and after (bottom) registration. From left to right: Patient cases 1-3, for point puncture (case 1), regional displacement (case 2), and two-sided contact (case 3).
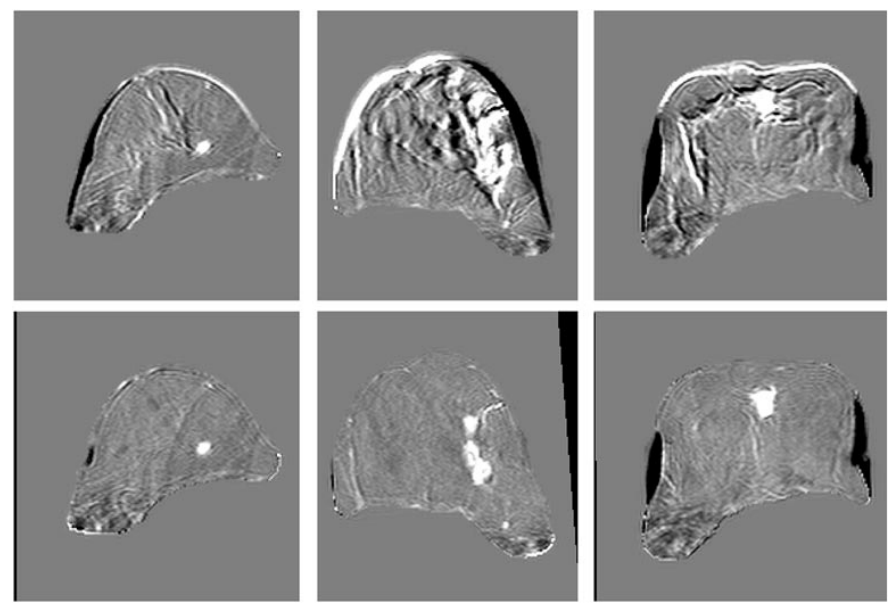

Fig. 7. Example subtraction slices of original precontrast images from example FEM deformation simulations before (top) and after (bottom) registration. (from left to right) Patient cases 1-3, for point puncture (case 1), regional displacement (case 2), and two-sided contact (case 3). Compare with original subtraction slices in Fig. 1.
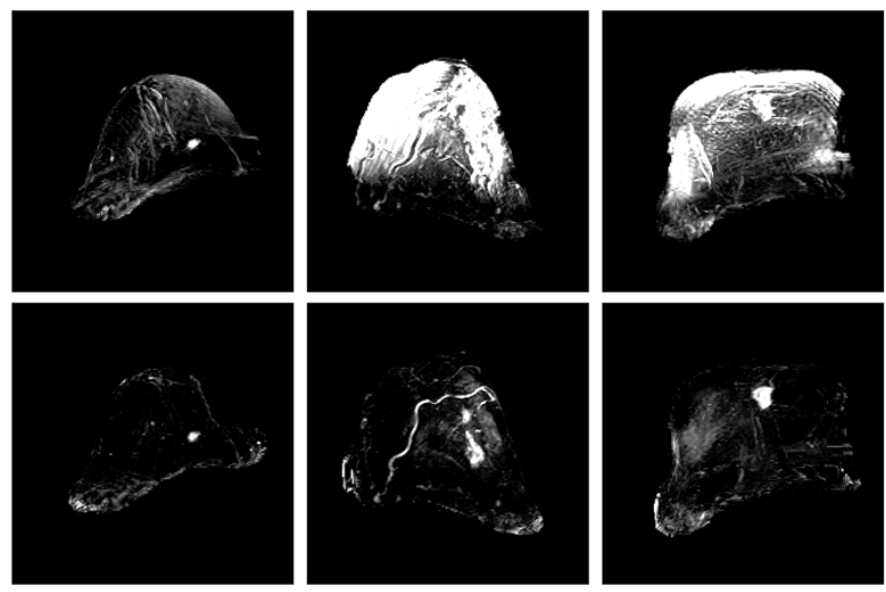

Fig. 8. MIPs through subtraction volumes of original precontrast images from example FEM deformation simulations before (top) and after (bottom) registration. (from left to right) Patient cases 1-3, for point puncture (case 1), regional displacement (case 2), and two-sided contact (case 3). Compare with original MIPs in Fig. 1. 

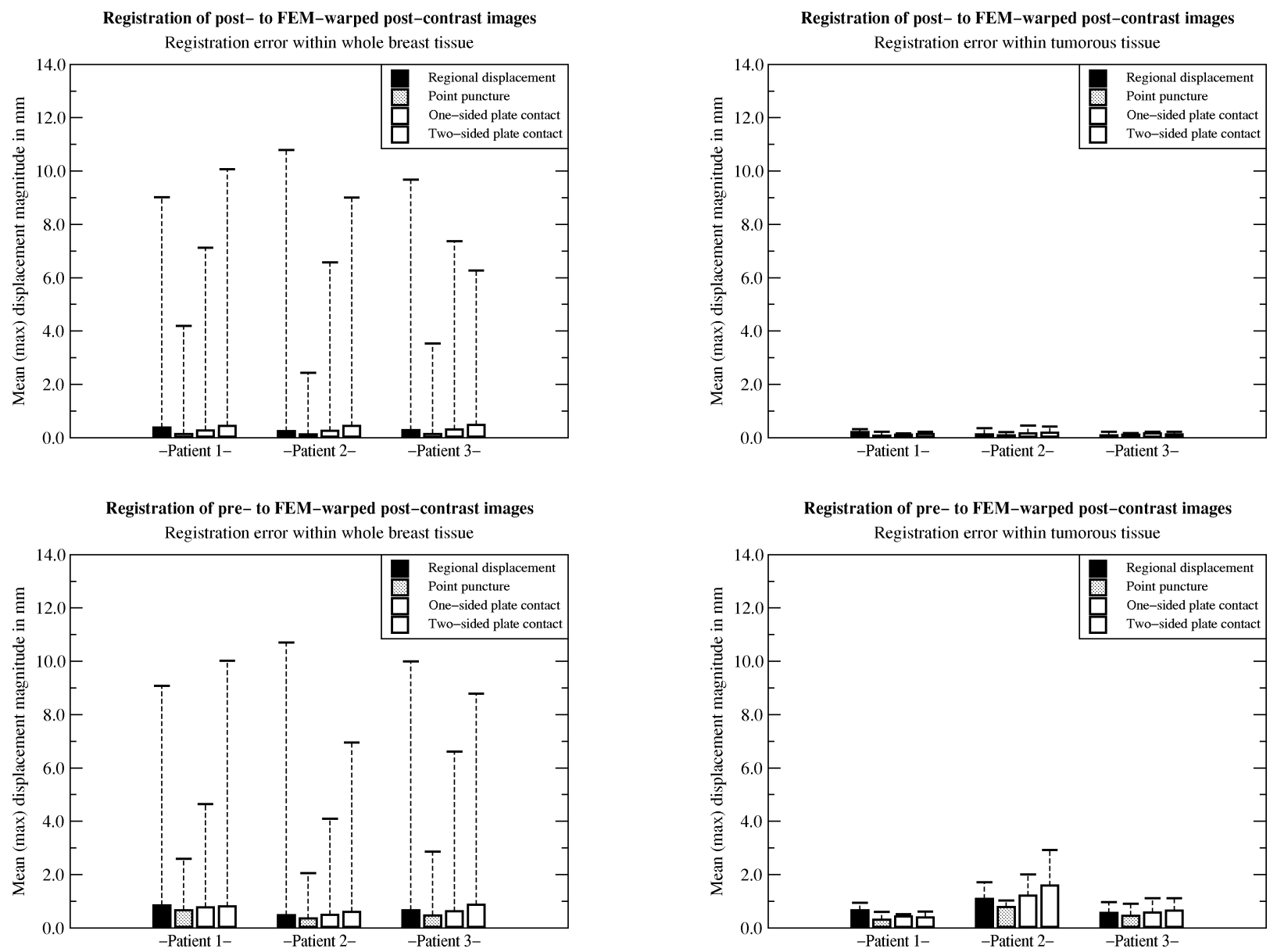

Fig. 9. Mean and maximum registration error for the registration of the original image pairs to FEM deformation simulations for the three patient cases, computed as mean voxel displacements in the whole breast volume (left) and in tumorous tissue (right). (top) registration of original postcontrast images to FEM deformed postcontrast images. (bottom) Registration of original precontrast images to FEM deformed postcontrast images.

MIPs through the subtraction volumes before and after registration. Although the registered subtraction images and MIPs are not directly comparable to the original subtraction images and MIPs in Fig. 1, as they are defined in the coordinate system of the FEM deformed images rather than the original images, they appear to be of a similar quality.

The bottom row of Fig. 9 shows the residual average and maximum registration errors for the precontrast image registration according to (5). The average registration error is slightly higher $(0.85 \mathrm{~mm})$ than for the postcontrast image registration described in Section V-B, with maximum errors between $2.86 \mathrm{~mm}$ and up to $10 \mathrm{~mm}$, which were also found to be localized outliers near the skin surface. Within the tumorous tissue, the average error within the tumors is slightly higher than in the whole breast tissue $(0.97 \mathrm{~mm})$, and the maximum errors are between $0.53 \mathrm{~mm}$ and $2.93 \mathrm{~mm}$, with the larger errors mainly occurring for patient 2 where the tumor lies close to the displaced skin surface. This higher error in the tumor region may also be an indication for the hypothesis that in CE image pairs, tumor volume is not sufficiently preserved by the nonrigid registration algorithm [11]. This may result in a very localized misregistration which now can be quantified with the presented validation methodology.

In summary, the registration accuracy for the registration method investigated using FEM simulations is slightly higher when registering the postcontrast image volumes to a FEM deformed version of themselves, than for registration of the precontrast to the FEM deformed postcontrast images. This is not surprising as there may remain a small amount of patient motion between the original, undeformed image pairs, which should be recovered by the registration and, thus, is quantified as a registration error. Both registration scenarios show very localized maximum errors at the skin surface. This error may be due to the masking of the FEM deformed breast images, leading to a local, sharp discontinuity of the simulated deformation field (in particular for the plate contact solutions). Smooth deformation interpolants such as the B-spline interpolation in the registration used in this paper may not compensate for this at the chosen control point resolution.

\section{DISCUSSION}

\section{A. Summary}

We have presented a novel and generic validation methodology for nonrigid medical image registration. This methodology is based on biomechanical tissue modeling using FEMs and simulations of gold standard deformations of tissue likely to occur during a real dynamic image acquisition. It assesses the registration accuracy quantitatively by comparing motion recovered by registration with the simulated tissue deformation at a voxel by voxel level. When testing a registration method over a 
range of data, based on different patient anatomies and deformation simulations, the validation framework provides the means to establish success or failure of a registration method for expected reasonable clinical input.

We have demonstrated the functionality of the presented validation methodology for an exemplar application using three CE MR mammographic image pairs, and a previously described nonrigid registration algorithm developed for that application [15]. The original image pairs were selected from a large database as being those few cases with negligible motion. This meant that patient motion during a dynamic image acquisition could be simulated by deforming the $\mathrm{CE}$ images only. The average accuracy of registering the original image pairs to the deformed CE images was found to be subvoxel using the exemplar registration method. Localized registration failures with higher registration errors could also be quantified by the validation method.

\section{B. Outlook}

The validation methodology presented here has scope for further improvement and extension. For more exhaustive deformation simulation and generation of data for nonrigid registration validation, the type, magnitude and locality of the FEM boundary conditions could be further varied, along with the material properties. For example, the models constructed in this paper treat all breast tissue as linear, which only holds for strains of less than $1 \%$ [28]. Instead, nonlinear elastic behavior of fibroglandular, ductile and cancerous tissue could be modeled [37]. Incorporating other important structures like the fibrous strands called Cooper's ligaments and the dynamic flexion and relaxation characteristics of the pectoral muscle is a challenging task which to our knowledge has not been adequately solved. Azar et al. [24] model the effect rather than the structure of Cooper's ligaments by nonlinearly increasing the stiffness of the fatty tissue, but this does not take the anisotropic ligament structure into account. In [38], they propose to model the pectoral muscle as the interface between breast and rib cage, allowing the nodes which are part of the breast tissue in contact with the rib cage to slide against the nodes representing the rib cage.

The use of a model-based gold standard simulation system as proposed in this paper may raise concern about the accuracy of the model and simulations themselves. In this paper, a generic image class for registration validation is simulated. More realistic, patient-specific modeling for predicting or emulating in vivo tissue deformations for a specific patient would require accurate and complete knowledge of the true boundary conditions and patient-specific tissue characteristics. Such knowledge, however, is quite difficult to obtain, and may even require the use of a registration method to establish correspondence. We recently conducted a case study for the comparison of different breast models of volunteer data deformed in vivo [39], including nonlinear models [24], [25], for a range of mesh resolutions, FEM solvers, Young's moduli and Poisson's ratios. The main conclusion from that work is that inaccurate assumptions of boundary conditions appear to have a much larger impact on the solutions than the chosen model parameters. Recently, Azar et al. [38] investigated the choice of material properties amongst other potential sources of error, and concluded that a model with very simplified material properties would not significantly affect the results for a given patient.

Finally, in on-going work [40], [41] we are applying the presented validation method to validate, compare and further improve the nonrigid registration algorithm by Rueckert et al. [15] and our recent extensions [42] with respect to local volume conservation and intrinsic registration parameters such as the choice of the control point mesh resolution.

\section{Conclusions}

The validation framework presented in this paper presents a generic approach for simulating tissue motion under the assumption of a specific tissue model. This can be adapted to patient-specific anatomy, FEM material properties and boundary conditions. The validation methodology we present here is a generic tool for generating images encompassing a range of physically plausible tissue deformations with which to test the accuracy and reliability of nonrigid registration algorithms. The methodology is not restricted to the exemplar nonrigid registration algorithm by Rueckert $e t$ al. [15] tested in this paper, but can be readily used for other nonrigid registration methods. Furthermore, the validation methodology is applicable to a wider range of intrasubject medical image registration problems where corresponding anatomy and tissue motion can be modeled using FEMs, such as brain, liver or cardiac applications. Finally, the validation methodology presented here could be used to help improve any nonrigid registration algorithm, or to compare the registration performance of a set of nonrigid registration methods.

\section{ACKNOWLEDGMENT}

The authors would like to thank Prof. F. A. Gerritsen and M. Quist from EasyVision Advanced Development, Medical Imaging Information Technology (MIMIT), Philips Medical Systems, Best, The Netherlands, and Dr. D. Rueckert from Imperial College London, United Kingdom, for useful discussions. They would also like to thank Dr. L. Sonoda from CISG, Dr. E. Denton, and Dr. S. Rankin from Guy's Hospital for access to the image database. Finally, they would like to thank Dr. P. Batchelor from CISG and J. Penrose from the University of Sheffield for their help in the model construction.

\section{REFERENCES}

[1] L. G. Brown, "A survey of image registration techniques," $A C M$ Comput. Surveys, vol. 24, no. 4, pp. 325-376, 1992.

[2] P. A. Van den Elsen, E.-J. D. Pol, and M. A. Viergever, "Medical image matching: A review with classification," IEEE Eng. Med. Biol. Mag., vol. 12, pp. 26-39, 1993

[3] J. B. A. Maintz and M. A. Viergever, "A survey of medical image registration," Med. Image Anal., vol. 2, no. 1, pp. 1-36, 1998

[4] H. Lester and S. R. Arridge, "A survey of hierarchical nonlinear medical image registration," Pattern Recogn., vol. 32, no. 1, pp. 129-149, 1999.

[5] Med. Image Registration, J. V. Hajnal, D. L. G. Hill, and D. J. Hawkes, Eds., CRC Press, 2001.

[6] J. M. Fitzpatrick, "Detecting failure, assessing success," in Med. Image Registration, J. V. Hajnal, D. L. G. Hill, and D. J. E. Hawkes, Eds: CRC Press, 2001, ch. I-6, pp. 117-139.

[7] C. Studholme, D. L. G. Hill, and D. J. Hawkes, "Automated 3D registration of MR and PET brain images by multi-resolution optimization of voxel similarity measures," Med. Phys., vol. 24, pp. 25-35, 1999.

[8] M. Holden, D. L. G. Hill, E. R. E. Denton, J. M. Jarosz, T. C. S. Cox, T. Rohlfing, J. Goodey, and D. J. Hawkes, "Voxel similarity measures for 3-D serial MR brain image registration," IEEE Trans. Med. Imag., vol. 19, pp. 94-102, Feb. 2000 
[9] J. M. Fitzpatrick, D. L. G. Hill, Y. Shyr, J. West, C. Studholme, and C. R. Maurer Jr., "Visual assessment of the accuracy of retrospective registration of MR and CT images of the brain," IEEE Trans. Med. Imag., vol. 17, pp. 571-585, Aug. 1998

[10] E. R. E. Denton, L. I. Sonoda, D. Rueckert, S. C. Rankin, C. Hayes, M. Leach, D. L. G. Hill, and D. J. Hawkes, "Comparison and evaluation of rigid and nonrigid registration of breast MR images," J. Comput. Assist. Tomogr., vol. 23, no. 5, pp. 800-805, 1999.

[11] C. Tanner, J. A. Schnabel, D. Chung, M. J. Clarkson, D. Rueckert, D. L. G. Hill, and D. J. Hawkes, "Volume and shape preservation of enhancing lesions when applying nonrigid registration to a time series of contrast enhancing MR breast images," in Lecture Notes in Computer Science. Berlin, Germany: Springer-Verlag, 2000, vol. 1935, Proc. Medical Image Computing and Computer-Assisted Intervention, pp. 327-337.

[12] J. B. West, J. M. Fitzpatrick, M. Y. Wang, B. M. Dawant, C. R. Maurer Jr., R. M. Kessler, R. J. Maciunas, C. Barillot, D. Lemoine, A. Collignon, F. Maes, P. Suetens, D. Vandermeulen, P. A. van den Elsen, S. Napel, T. S. Sumanaweera, B. Harkness, P. F. Hemler, D. L. G. Hill, D. J. Hawkes, C. Studholme, J. B. A. Maintz, M. A. Viergever, G. Malandain, X. Pennec, M. E. Noz, G. Q. Maguire Jr., M. Pollac, C. A. Pelizzari, R. A. Robb, D. Hanson, and R. P. Woods, "Comparison and evaluation of retrospective intermodality brain image registration techniques," $J$. Comput. Assist. Tomogr., vol. 21, no. 4, pp. 554-566, 1997.

[13] K. Rohr, M. Fornefett, and H. S. Stiehl, "Approximating thin-plate splines for elastic registration: Integration of landmark errors and orientation attributes," in Lecture Notes in Computer Science. Berlin, Germany: Springer-Verlag, 1999, vol. 1613, Proc. Information Processing in Medical Imaging, pp. 252-265.

[14] J. A. Schnabel, C. Tanner, A. D. Castellano-Smith, M. O. Leach, C. Hayes, A. Degenhard, R. Hose, D. L. G. Hill, and D. J. Hawkes, "Validation of nonrigid registration using finite element methods," in Lecture Notes in Computer Science. Berlin, Germany: Springer-Verlag, 2001, vol. 2082, Proc. International Conference on Information Processing in Medical Imaging, pp. 344-357.

[15] D. Rueckert, L. I. Sonoda, C. Hayes, D. L. G. Hill, M. O. Leach, and D. J. Hawkes, "Non-rigid registration using free-form deformations: Application to breast MR images," IEEE Trans. Med. Imag., vol. 18, pp. 712-721, Aug. 1999

[16] J. Z. Chen, S. M. Pizer, E. L. Chaney, and S. Joshi, "Medical image synthesis via Monte Carlo simulation," in Lecture Notes in Computer Science. Berlin, Germany: Springer-Verlag, 2002, vol. 2489-I, Proc. Medical Image Computing and Computer Assisted Intervention, pp. 347-354.

[17] D. Rueckert, A. F. Frangi, and J. A. Schnabel, "Automatic construction of 3D statistical deformation models using nonrigid registration," in Lecture Notes in Computer Science. Berlin, Germany: Springer-Verlag, 2001, vol. 2208, Proc. Medical Image Computing and Computer-Assisted Intervention, pp. 77-84.

[18] C. Studholme, D. L. G. Hill, and D. J. Hawkes, "An overlap entropy measure of 3D medical image alignment," Pattern Recogn., vol. 32, pp. $71-86,1999$.

[19] M. I. Miga, K. D. Paulsen, J. M. Lemery, S. D. Eisner, A. H. Hartov, F. E. Kennedy, and D. W. Roberts, "Model-updated image guidance: Initial clinical experiences with gravity-induced brain deformation," IEEE Trans. Med. Imag., vol. 18, pp. 866-874, Oct. 1999.

[20] M. Ferrant, S. K. Warfield, A. Nabavi, F. A. Jolesz, and R. Kikinis, "Registration of 3D intraoperative MR images of the brain using a finite element biomechanical model," in Lecture Notes in Computer Science. Berlin, Germany: Springer-Verlag, 2000, vol. 1935, Proc Medical Image Computing and Computer-Assisted Intervention, pp. 19-28.

[21] O. Škrinjar, C. Studholme, A. Nabavi, and J. Duncan, "Steps toward a stereo-camera-guided biomechanical model for brain shift compensation," in Lecture Notes in Computer Science. Berlin, Germany: Springer-Verlag, 2001, vol. 2082, Proc. Information Processing in Medical Imaging, pp. 183-189.

[22] A. Hagemann, K. Rohr, H. S. Stiehl, U. Spetzger, and J. M. Gilsbach, "Biomechanical modeling of the human head for physically based, nonrigid image registration," IEEE Trans. Med. Imag., vol. 18, pp. 875-884, Oct. 1999.
[23] A. Castellano-Smith, T. Hartkens, J. Schnabel, R. Hose, H. Liu, W. A. Hall, C. L. Truwit, D. J. Hawkes, and D. L. G. Hill, "Constructing patient specific models for correcting intraoperative brain deformation," in Lecture Notes in Computer Science. Berlin, Germany: Springer-Verlag, 2001, vol. 2208, Proc. Medical Image Computing and Computer-Assisted Intervention, pp. 1091-1098.

[24] F. S. Azar, D. N. Metaxas, and M. D. Schall, "A finite model of the breast for predicting mechanical deformations during biopsy procedure," in Proc. IEEE Workshop Mathematical Methods in Biomedical Image Analysis, 2000, pp. 38-45.

[25] A. Samani, J. Bishop, M. J. Yaffe, and D. B. Plewes, "Biomechanical 3-D finite-element modeling of the human breast using MRI data," IEEE Trans. Med. Imag., vol. 20, pp. 271-279, Apr. 2000.

[26] R. Sinkus, J. Lorenzen, D. Schrader, M. Lorenzen, M. Dargatz, and D. Holz, "High-resolution tensor MR elastography for breast tumour detection," Phys. Med. Biol., vol. 45, pp. 1649-1664, 2000.

[27] D. B. Plewes, J. Bishop, A. Samani, and J. Sciaretta, "Visualization and quantification of breast cancer biomechanical properties with magnetic resonance elastography," Phys. Med. Biol., vol. 45, pp. 1591-1610, 2000.

[28] M. M. Doyley, P. M. Meaney, and J. C. Bamber, "Evaluation of an iterative reconstruction method for quantitative elastography," Phys. Med. Biol., vol. 45, pp. 1521-1539, 2000.

[29] M. I. Miga, "A new approach to elastographic imaging: Modality independent elastography," in Proc. Medical Imaging 2002: Image Processing, vol. 4684, 2002, pp. 604-611.

[30] Biomedical Image Resource, Mayo Foundation, Rochester, MN.

[31] W. Schroeder, K. Martin, and B. Lorensen, The Visualization Toolkit, 2nd ed. Englewood Cliffs, NJ: Prentice-Hall, 1997.

[32] A. Sarvazyan, D. Goukassian, E. Maevsky, and G. Oranskaja, "Elastic imaging as a new modality of medical imaging for cancer detection," in Proc. Int Workshop Interaction of Ultrasound With Biological Media, 1994, pp. 69-81.

[33] J. B. Park, Biomaterials Science and Engineering. New York: Plenum, 1984.

[34] A. J. Davis, The Finite Element Method: A First Approach. Oxford, U.K.: Oxford Univ. Press, 1980.

[35] S. Lee, G. Wolberg, and S. Y. Shin, "Scattered data interpolation with multilevel B-splines," IEEE Trans. Visual. Comput. Graphics, vol. 3, pp. 228-244, July/Sept. 1997.

[36] R. W. Hamming, Digital Filters. Englewood Cliffs, NJ: Prentice-Hall, 1989.

[37] P. S. Wellman, "Tactile imaging," Ph.D. dissertation, Harvard Univ., Cambridge, MA, 1999.

[38] F. S. Azar, D. N. Metaxas, and M. D. Schall, "Methods for modeling and predicting mechanical deformations of the breast under external pertubations," Med. Image Anal., vol. 6, no. 1, pp. 1-27, 2002.

[39] C. Tanner, A. Degenhard, J. A. Schnabel, A. D. Castellano-Smith, C. Hayes, L. I. Sonoda, M. O. Leach, D. R. Hose, D. L. G. Hill, and D. J. Hawkes, "A comparison of biomechanical breast models: A case study," in Proc. Medical Imaging 2002: Image Processing, vol. 4684, 2002, pp. 1807-1818.

[40] C. Tanner, J. A. Schnabel, A. Degenhard, A. D. Castellano-Smith, C. Hayes, M. O. Leach, D. R. Hose, D. L. G. Hill, and D. J. Hawkes, "Validation of volume-preserving nonrigid registration: Application to contrast-enhanced MR-mammography," in Lecture Notes in Computer Science Berlin, Germany, 2002, vol. 2489-I, Proc. Medical Image Computing and Computer-Assisted Intervention, pp. 307-314.

[41] J. A. Schnabel, C. Tanner, A. D. Castellano-Smith, A. Degenhard, C. Hayes, M. O. Leach, D. R. Hose, D. L. G. Hill, and D. J. Hawkes, "Finite element based validation of nonrigid registration using singleand multi-level free-form deformations: Application to contrast-enhanced MR mammography," in Proc. Medical Imaging 2002: Image Processing, vol. 4684, 2002, pp. 550-561.

[42] J. A. Schnabel, D. Rueckert, M. Quist, J. M. Blackall, A. D. Castellano-Smith, T. Hartkens, G. P. Penney, W. A. Hall, H. Liu, C. L. Truwit, F. A. Gerritsen, D. L. G. Hill, and D. J. Hawkes, "A generic framework for nonrigid registration based on nonuniform multi-level free-form deformations," in Lecture Notes in Computer Science Berlin, Germany, 2001, vol. 2208, Proc. Medical Image Computing and Computer-Assisted Intervention, pp. 573-581. 